\title{
50 AÑOS DE VIDA DE LA UPOLI'
}

\section{Universidad cristiana- evangélica de probada vocación de servicio público, con talento y compromiso}

\author{
Recibido: 30/03/2017 / Aprobado: 31/03/2017
}

Por Sergio Denis García V.2

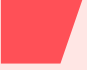

\section{Resumen}

Sergio Denis García, realiza en ocasión de los 50 años de fundación de la UPOLI, un breve recorrido de su trayectoria. Nos relata que la UPOLI fue la primera universidad cristiana evangélica en nuestro país y la segunda a nivel centroamericano. Siendo un proyecto educativo ideado y soñado por la Convención Bautista de Nicaragua (CBN). La UPOLI, en sus inicios (1967) se creó y funcionó como Instituto Politécnico de Nicaragua, el POLI. Posteriormente, mediante Decreto Legislativo, obtuvo rango de Universidad, y a lo largo de sus 50 años de vida, ha venido funcionando por convicción propia y designación estatal, como una universidad de servicio público, sin ánimo de lucro.

Algunos expertos la han reconocido como una universidad con vocación especial para servir las necesidades de los sectores de la juventud nicaragüense provenientes de las clases económicas sociales emergentes (medias y medias bajas). Su Lema 'Sirviendo a la Comunidad', la ha llevado a ser una de las instituciones de educación superior con más clara vocación de servicio.

Palabras clave: cristiana-evangélica, bautista, servicio, compromiso, vocación.

La Universidad Politécnica de Nicaragua (UPOLI), es la primera universidad cristiana evangélica no confesional que se fundó en el país, y la segunda a nivel centroamericano. Es la tercera universidad establecida en Nicaragua después de la UNAN y la UCA, y junto a estas dos universidades son las de mayor trayectoria y que han persistido en el tiempo, alcanzado la privilegiada edad de 50 o más años de funcionamiento exitoso.
2017, es entonces el año del 50 Aniversario de Fundación de la UPOLI, un momento cumbre de triunfo y de gloria que evoca una jornada de fe, de perseverancia, de lucha inclaudicable de fundadores y continuadores de la Obra, de acción de gracias y reconocimiento de la presencia de Dios en todo momento, de una trayectoria luminosa y trascendente de aportes de gran provecho para el pueblo nicaragüense.

1 Versión ampliada del Editorial publicado en Cultura de Paz. Managua, Nicaragua. Volumen 23, № 71. Enero-Abril 2017.

2 Rector de UPOLI (1980-2003), Rector Emérito, Miembro del Patronato Universitario UPOLI. 
En 1917 surge la Obra Bautista en la zona del Pacífico de Nicaragua, atendiendo la predicación del Evangelio de Jesucristo, pero también las necesidades comunitarias, especialmente en lo referente a la salud y educación. En este año 2017 se celebra el Centenario de la Obra Bautista.

Aunque no hay testimonios explícitos, se conoce que en el pueblo bautista siempre estuvo latente por muchos años la idea de contar con una universidad propia de esta denominación evangélica.

Pastores de la Iglesia Bautista, previo al nacimiento de la universidad, impulsaron trabajos de reflexión con universitarios evangélicos, como los realizados en León, Ciudad Universitaria, por los pastores, primero el Rev. Heriberto Vásquez y luego por el Rev. Oscar Espino. En esos tiempos se formó el Frente Evangélico de Universitarios Nicaragüenses (FEDUN). Estos esfuerzos fueron respaldados con la relación que se estableció con la Federación Mundial Cristiana de Estudiantes, con sede en Ginebra, Suiza, que más tarde dio origen al Movimiento Estudiantil Cristiano (MEC) de Nicaragua, que realizó actividades consideradas como "Antecedentes Fundacionales Inmediatos de la UPOLI". ${ }^{3}$

\section{El Comité Pro-universidad Bautista}

Como dijimos, esta idea e interés de fundar una universidad Bautista, estuvo latente por varios años y dio lugar a la formación del Comité Pro-Universidad Bautista que estaba integrado por el Dr. y Rev. Rolando Gutiérrez Cortés, Pastor de la Primera Iglesia Bautista de Managua; el Profesor Gustavo Wilson B., Director del Colegio Bautista; y el Dr. Norberto Herrera Z., en ese entonces profesor y administrador de la Escuela de Ciencias de la Educación de la UNAN (Universidad Nacional Autónoma de Nicaragua), personajes sobresalientes poseídos de gran carisma y de entusiasmo por el proyecto, y que más tarde integrarían el Patronato Fundador de la UPOLI.

El Dr. Herrera además llegó a ser el primer Rector de la universidad. Más tarde la idea y sueño de la universidad Bautista se trasladó al seno de la Convención Bautista de Nicaragua (CBN), donde la "idea universitaria" empieza a configurarse como un proyecto. En abril de 1966, en la ciudad de León, en la XXX Asamblea General de la CBN se oficializó el Comité ProUniversidad Bautista. El 23 de mayo de 1966, este Comité con la participación destacada del Dr. Herrera, preparó el documento "Pensando en la Universidad Bautista", en el cual se definía la filosofía que adoptaría el proyecto educativo.

\section{Creación de la UPOLI y del Patronato Fundador}

En el año 1967 siendo presidente de la CBN el Dr. Norberto Herrera, en la XXXI Asamblea General realizada en Managua del 25 de febrero al 06 de marzo, se aprobó con beneplácito el informe de la Comisión ProUniversidad Bautista que incluía el aspecto fundacional, así como la lista de los 15 miembros que se propuso constituyeran el Patronato Fundador de la UPOLI, con carácter permanente. "También se aprobó el carácter autónomo de la Universidad Bautista, por cuanto facultaba al Patronato a que, en base al principio de la autonomía universitaria, fuera el responsable de realizar todas las gestiones necesarias para que la Universidad Bautista se hiciera realidad", expresa el Dr. Norberto Herrera Z., Rector Fundador de la UPOLI, en su obra Vale la Pena Vivir, editada en el año 2010.

Como ya se expresó, en el documento que elaboró el Comité Pro-Universidad Bautista que la CBN aprobó

3 Sembrando con lágrimas... Cosechando con regocijo. La UPOLI: 40 años sirviendo a la comunidad. Managua. UPOLI, 2007. 360P. 
en el año 1967, incluía todo lo relativo a los aspectos fundacionales de la Universidad Bautista, que luego por circunstancias se denominaría Universidad Politécnica de Nicaragua, (UPOLI), de tal manera que en este evento y fecha (entre el 25 de febrero y el 06 de marzo del año 1967, se dio propiamente la creación oficial de la UPOLI por parte de la CBN).

Se creó también oficialmente el Patronato Fundador Pro-Universidad Bautista, cuya constitución legal se dio el 1 de marzo de ese año, a través de la firma de su Acta Constitutiva.

En el Acta se definió entre otras cosas, que el Patronato sería el responsable de "efectuar las tareas que conduzcan a la pronta obtención de la autorización necesaria para que funcione el instituto. (La UPOLI en sus inicios se creó y funcionó como Instituto Politécnico de Nicaragua, el POLI). Esta ansiada y necesaria autorización de funcionamiento se dio el 25 de noviembre del año 1967, mediante el Decreto Ejecutivo No. 76-J, firmado por el Presidente de la República y el Ministro de Educación de ese entonces. En 1977 mediante Decreto Legislativo, la institución obtuvo rango de Universidad, el POLI pasó a ser la UPOLI.

Sorprendentemente esta fecha del 25 de noviembre del año 1967 es la que se acuñó como la fecha

de fundación de la UPOLI, en vez de la que correspondió entre el 25 de febrero y el 06 de marzo de ese mismo año, que fue cuando la CBN aprobó la creación de la Universidad Bautista.

El Acta Constitutiva definió al POLI como una "institución privada", al Patronato, como una asociación "con fines culturales y sin ánimo de lucro" y a los miembros del Patronato como "bautistas interesados en el desarrollo económico, social y cultural de los nicaragüenses".

Una vez que se aprobaron los Estatutos, el POLI quedó establecido como "Una institución privada de educación superior, de motivación cristiana evangélica y con carácter de servicio público".

En el documento "Pensando en la Universidad Bautista", se concibió la universidad como una "institución de alto nivel académico que testifique a Jesucristo por medio del 'Servicio a la Comunidad', utilizando el lema "Sirviendo a la Comunidad" que sustenta la UPOLI hasta la fecha.

Por una decisión reprochable y discriminatoria, el Presidente de la República de la época "dijo en un acto típicamente dictatorial - expresa el Dr. Herrera en su Obra Vale la Pena Vivir -, aprobaremos esto hoy mismo" por Decreto Ejecutivo, pero para no pelearme con mi primo (Rector en ese tiempo de la Universidad Jesuita, UCA), le cambiaremos el nombre y le llamaremos Instituto Politécnico de Nicaragua. Todo lo demás, fuera del nombre, quedará tal como ustedes lo solicitan..."

\section{Probada vocación educativa de servicio público}

La UPOLI, como ya se mencionó es una institución privada, de educación superior, y por convicción propia y designación estatal ha funcionado a lo largo de

En primer plano, de izquierda a derecha: Profesor Roberto López, ex Director de Colegio Bautista; Rev. José Miguel Torres, Pastor y Teólogo Ecuménico y Dr. y Rev. Rolando Gutiérrez Cortés, Presidente del Comité Pro Universidad, Pastor de la Primera Iglesia Bautista de Managua; entonces dirigentes de las juventudes bautistas de Nicaragua. sus 50 años de vida, como una universidad de servicio público, sin ánimo delucro. Por convicción propia, debido 


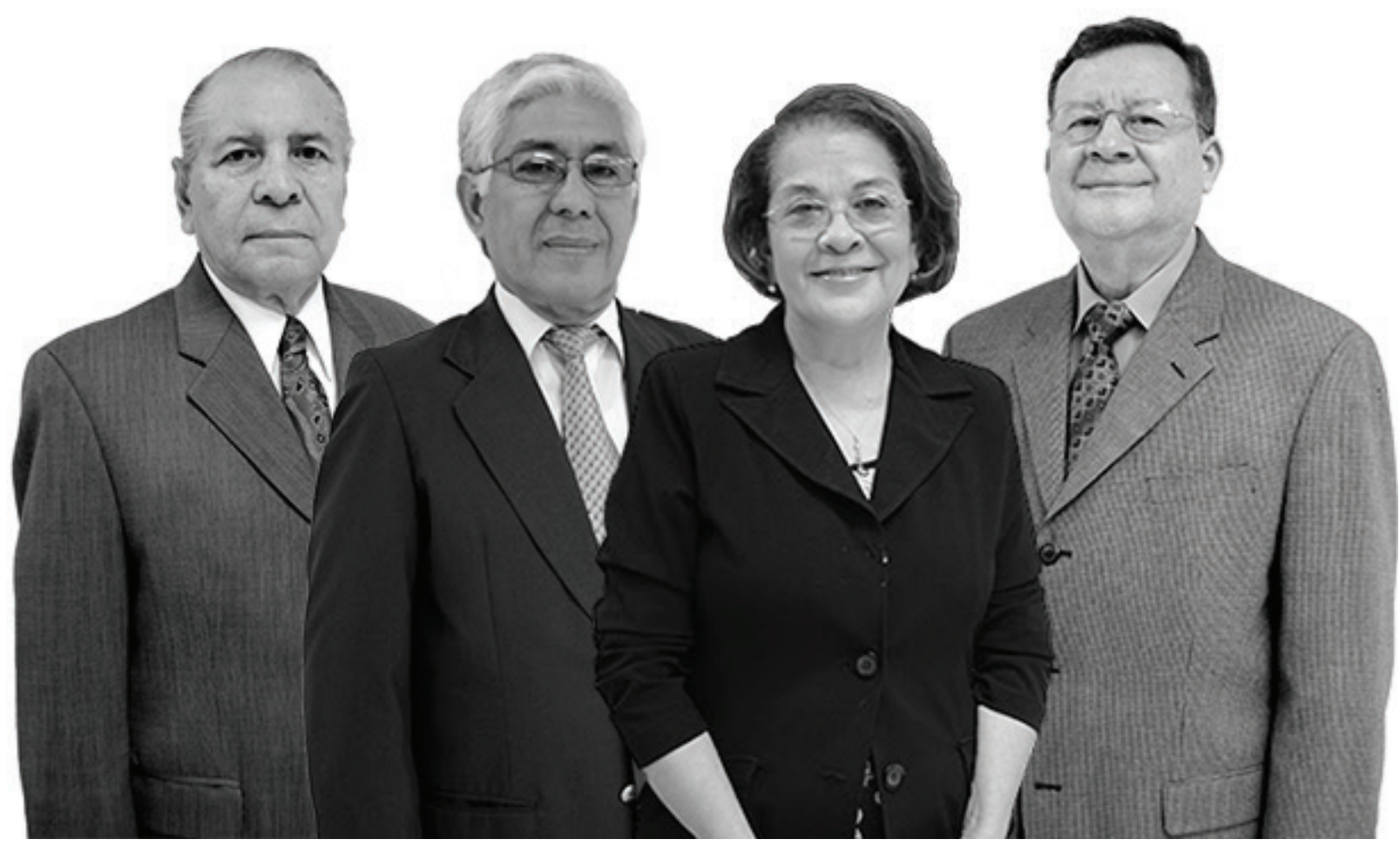

Rectores a lo largo de los 50 años de la UPOLI. De izquierda a derecha: Dr. Norberto Herrera, Rector Fundador (1967-1979); Ing. Emerson Pérez Sandoval (2004-2012); Dra. Lydia Ruth Zamora (2013 a la actualidad) y Dr. Sergio Denis García, Rector Emérito (1980-2003).

a su motivación cristiana evangélica, la UPOLI ha tratado de responder y ser fiel a la voluntad de sus fundadores, preservando la visión y el énfasis con que fue concebida desde su creación, confirmando su propósito y misión, procurando una formación educativa integral y con valores cristianos en sus estudiantes. Los consultores Dr. Carlos Tünnermann e Ing. Miguel E. Vijil, en su trabajo: "Diagnóstico Situacional de la UPOLI", elaborado en el año 2001, expresaban que", ... a la UPOLI también se le reconoce como una universidad con vocación especial para servir las necesidades de los sectores de la juventud nicaragüense provenientes de las clases económicas sociales emergentes (medias y medias bajas). Agregaban: Su Lema 'Sirviendo a la Comunidad', la ha llevado a ser una de las instituciones de educación superior con más clara vocación de servicio (...). “El Dr. Norberto Herrera, Rector Fundador de la UPOLI, en carta al Rev. Valdo Galland (de la Iglesia Valdense de Suiza), año 1968, expresaba: "Nuestro POLI es una universidad, que ofrece alternativas de formación profesional a sectores emergentes...".

Por designación estatal. En el año 1980 durante el Gobierno Revolucionario en su primer momento, la UPOLI fue una de las universidades reconocidas por su calidad y trayectoria académica, y pasó a ser parte de la oferta educativa de nivel superior de servicio público del país, mediante subvención económica estatal del $100 \%$, por la gratuidad establecida en ese tiempo y que duró hasta el año 1990. A partir de este año la UPOLI ha funcionado en su presupuesto con aporte económico estatal y con ingresos propios, por lo cual hay estudiantes con subvención total y otros subvencionados parcialmente, en este último caso, estos gozan comparativamente hablando, del pago de los aranceles más bajos de la Educación Superior del país, con respecto a otras universidades de calidad académica equivalente.

\section{Talento y Experiencia Educativa}

En la obra Historia de los 40 Años de la UPOLI, se refiere que existen iniciativas y proyectos historiográficos que aportan información importante para comprender lo arraigado de la vocación y trayectoria de servicio comunitario, particularmente del servicio educativo, de los bautistas de Nicaragua, en su estrategia misionera. En el transcurso de un siglo, la obra Bautista se ha caracterizado y se reconoce porque además de la 


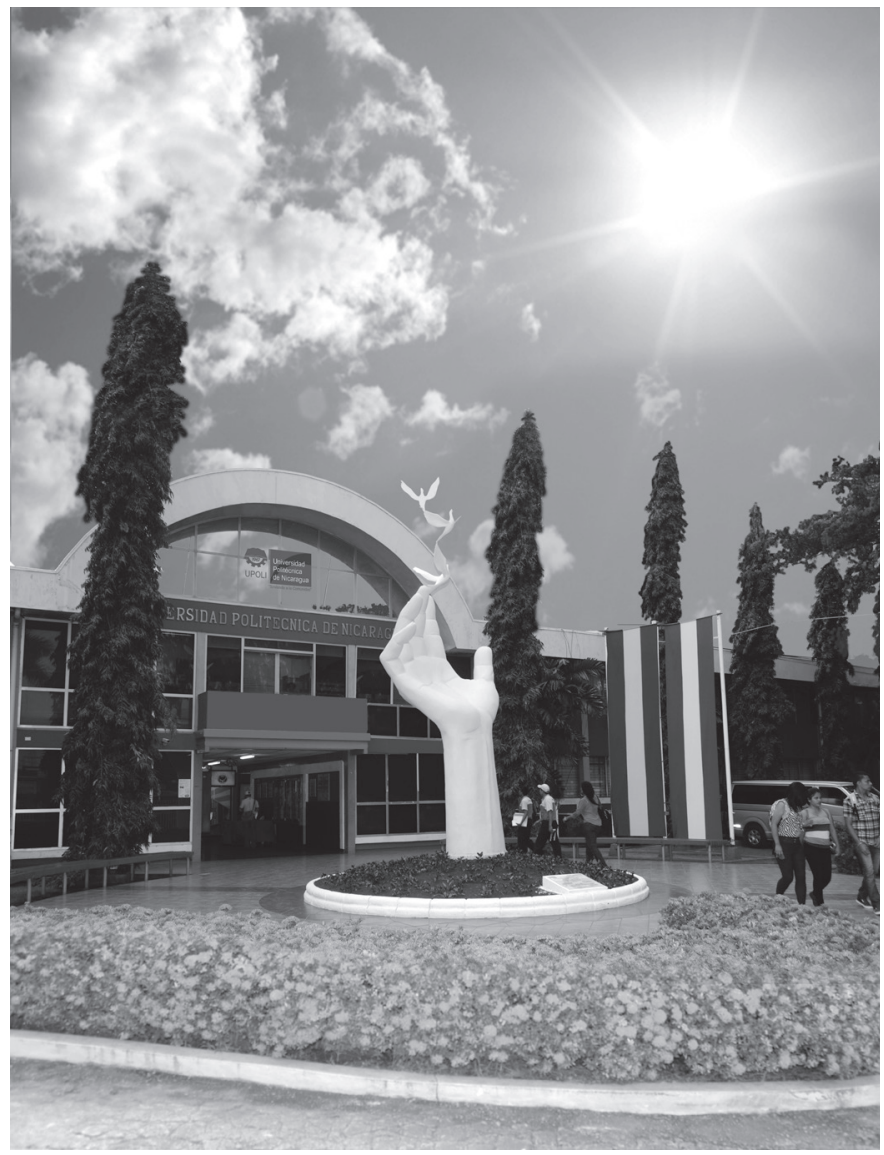

predicación del Evangelio, la organización de Iglesias y Congregaciones, se ha dado a la creación de instituciones educativas, del área de la salud y asistencia social de gran prestigio. En cuanto la atención educativa, estas importantes iniciativas se han dado desde mediados del siglo XIX en el Caribe (Costa Atlántica), e inicios del siglo XX en la región del Pacífico, como un medio para mejorar las condiciones de vida de la población, y combatir los males que les afectan.

El talento y trayectoria de servicio educativo de calidad, y apropiado a las necesidades del país, es algo que desarrollaron con éxito y heredaron los iniciadores de la Obra Bautista a los líderes fundadores de la UPOLI. La Historia de los 40 años de la UPOLI aborda con amplitud este tema:

Había conciencia entre los quince miembros del Patronato Fundador del POLI que el mejor aporte que podían hacer los bautistas era en el área de la educación tecnológica. Los quince miembros representaban, hasta cierto punto, esa clase media pujante que, viniendo desde abajo y superando obstáculos, se insertaba con éxito y prestigio en la sociedad civil de aquel momento.

"Entonces, frente a las carreras tradicionales de la época (...) se sintió la necesidad de formar cuadros técnicos que reforzaran el aparataje de las necesidades sociales emergentes.

“... Las primeras carreras que abre la UPOLI fueron las de Asistentes Ejecutivos, Dibujo Publicitario, Electrónica, Educación Física, Decoración de Interiores, Diseño y Enfermería". El Dr. Norberto Herrera en su obra Vale la Pena Vivir, refiere que cuando se abordó al Presidente de la República de la época, sobre la autorización de funcionamiento de la UPOLI, lo que más le llamó la atención al Presidente fue que el énfasis estaría en carreras tecnológicas y no las asociadas a las artes liberales tradicionales.

Esta opción y diseño de carreras apropiadas a la realidad y necesidad del país, en los inicios de vida de la universidad, es una prueba entre muchas más, del talento y la capacidad del liderazgo que fundó la UPOLI, que supo calibrar el momento y proponer una alternativa viable, provechosa para la vida del país.

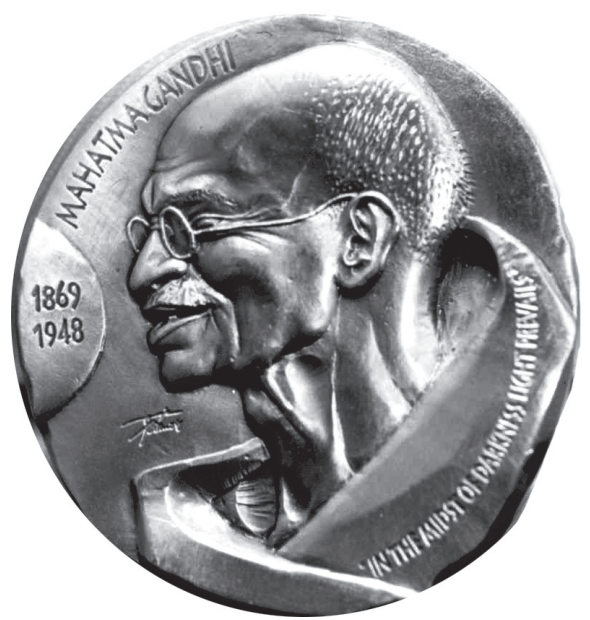

Medalla Mahatma Gandhi otorgada por la UNESCO en 1997, al Dr. Sergio Denis García, entonces Rector de UPOLI y al MSc. Denis Torres, Director del IMLK-UPOLI, por su incansable lucha en favor de los Derechos Humanos y la promoción de una Cultura de Paz. La más alta distinción recibida por la UPOLI en sus 50 años. 


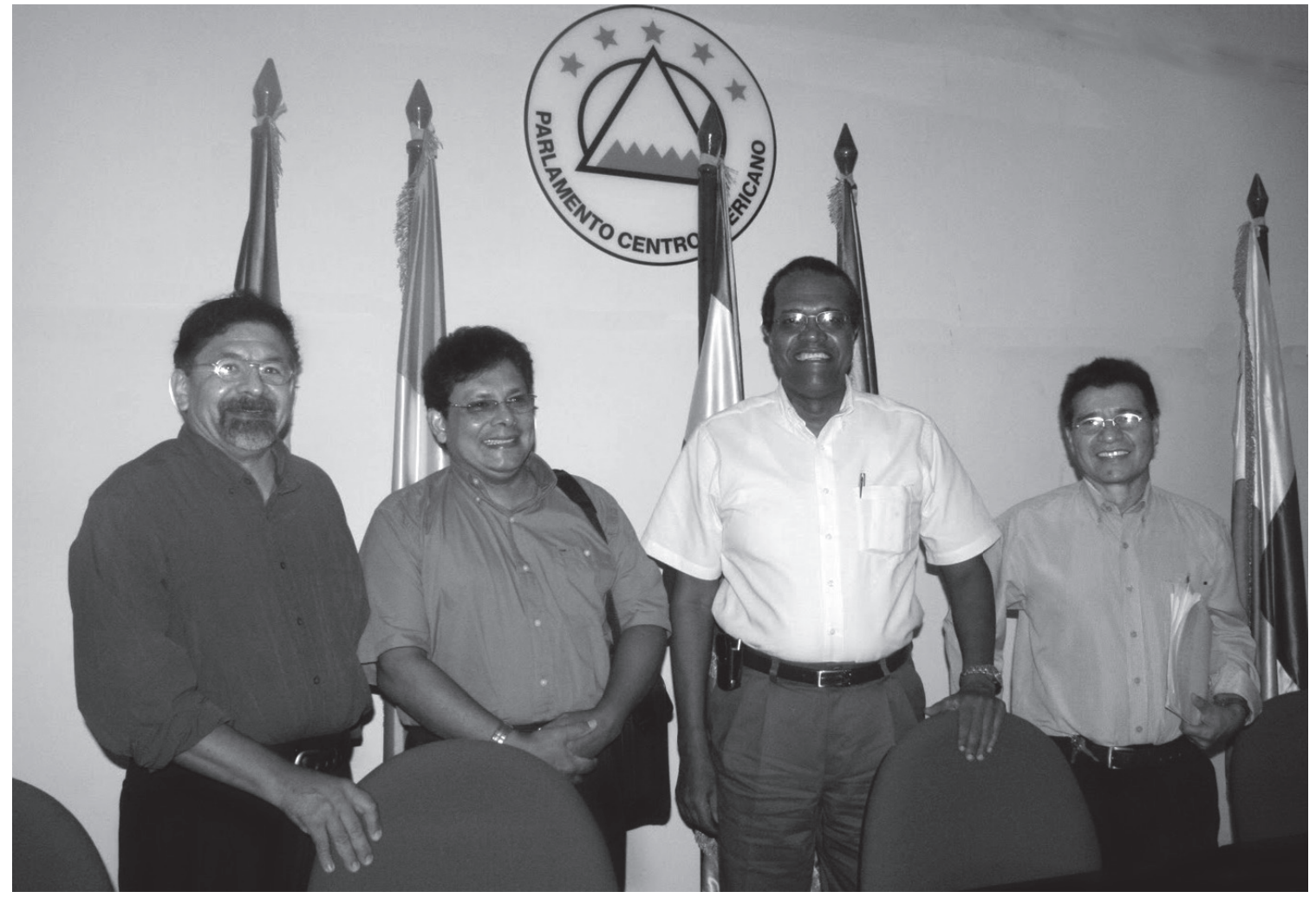

Rev. José Miguel Torres (q.e.p.d), pacifista, pastor y teólogo ecuménico; MSc. Denis Torres, Director del IMLK; Dr. Francisco Campbell, entonces Vicepresidente del PARLACEN y el MSc. Ezequiel Montenegro, Responsable de Relaciones Internacionales del IMLK, en la Presentación de la Iniciativa "Centroamérica: Zona de Paz, Cooperación y Seguridad Ciudadana" en el PARLACEN, aprobada en el 2007.

Otra mención relevante del talento, y capacidad del liderazgo de la UPOLI es la que se menciona en la obra La Universidad Hacia Nuevos Horizontes (septiembre de 1993), escrita por el eminente educador, Dr. Carlos Tünnermann B., quien refiere lo siguiente:

Una de las singularidades de la UPOLI, en sus primeros veinticinco años de existencia, es que su comunidad universitaria (autoridades, profesores y estudiantes) han optado la concepción de que la UPOLI es un 'Proyecto Educativo' con características muy propias y objetivos lúcidamente definidos: una universidad no estatal de servicio público y raíz cristiana, que sirve a la comunidad mediante el ofrecimiento de posibilidades de formación superior y universitaria a sectores sociales emergentes.

De igual manera el Dr. Tünnermann refiere en esta obra,... que otro aspecto que distingue y caracteriza a la UPOLI fue su perfil de carreras a desarrollarse en dos fases, una de Técnico Superior, y una complementaria para completar licenciatura, creando una "salida lateral" natural al mundo del trabajo (después del Técnico Superior), y la posibilidad de reingreso para alcanzar niveles académicos superiores, algo que el Dr. Tünnermann calificó, que coincide con una de las recomendaciones de los especialistas que por encargo de la UNESCO estaban empeñados en reflexionar sobre la educación superior en el Siglo XXI.

\section{Compromiso}

Hemos venido resaltando a lo largo de este escrito, la probada vocación de servicio público de la UPOLI como universidad cristiana evangélica, Bautista para más precisión, demostrando y poniendo en evidencia la capacidad y talento en el desempeño,... pero además, ha sido visible y manifiesto una característica relevante que ha estado presente siempre, y es su compromiso y empeño de atención y servicio de los sectores más 

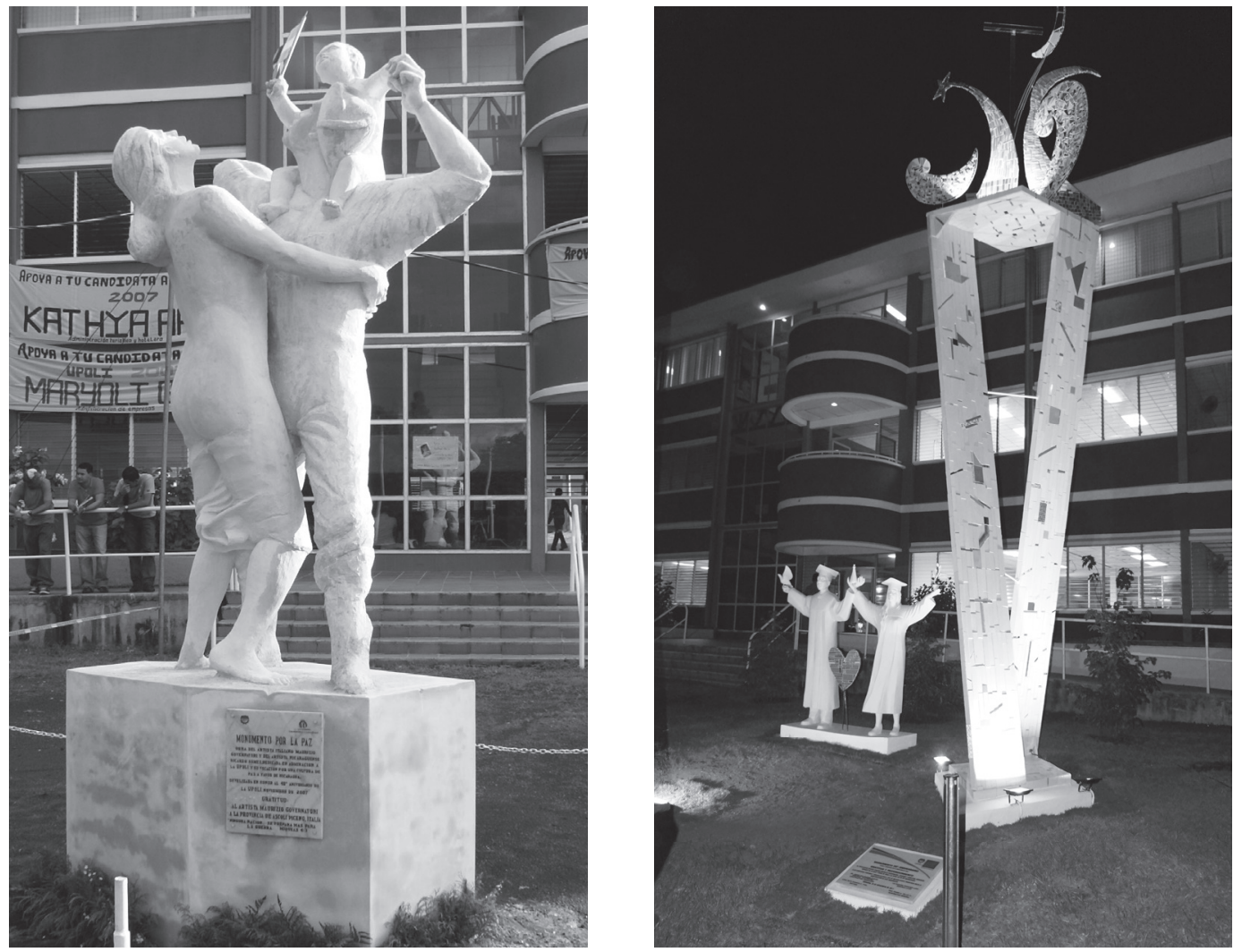

Obras del Maestro Maurizio Governatori, escultor y muralista italiano. La primera se titula "La paz y la familia" y la segunda "Monumento del 50 aniversario de la UPOLI". Éstas y otras obras se encuentran en el Campus Universitario de la UPOLI. Foto: Edward Dávila/ Relaciones Públicas UPOLI.

necesitados del país. En la obra de los 40 años de Historia de la UPOLI se hace referencia a lo expresado por el Dr. Jorge Pixley, destacado teólogo biblista, en su obra Por una Iglesia Laica, año 1999, quien afirma que:

Los bautistas, por su parte, eran gente de procedencia humilde, pero que veían en el trabajo y la educación los medios para mejorar su condición de vida, (...), pero frente a esta realidad estaba la firme determinación de servir educación superior a sectores emergentes de la población.

Esta motivación y compromiso se puso en evidencia por el hecho mismo de haber ubicado su campus central después del terremoto de 1972, en barrios pobres y marginados al oriente de Managua, la capital, demostrativo de esta opción de los fundadores de la UPOLI, a favor de sectores poblacionales limitados económicamente.

Ya se mencionó antes, que los quince miembros del Patronato fundador representaban a la clase media del país, que "viniendo desde abajo y superando obstáculos, se insertaban con éxito y prestigio en la sociedad civil de aquel momento", una clara manifestación de su visión y puesta en práctica de su lema "Sirviendo a la Comunidad". El Dr. Norberto Herrera, Rector Fundador de la UPOLI, en su obra Vale la Pena Vivir, expresó algo muy solemne y contundente que debe prevalecer: "Nuestro compromiso como universidad no lucrativa y de servicio público, es indeclinable. Nuestro compromiso es con nuestro amado pueblo".

Honra y gloria a los fundadores de la UPOLI que nos antecedieron, que han dejado una huella indeleble en la historia de nuestra universidad, ya que con su visión, esfuerzo y ejemplo labraron los cimientos de una Institución firme y robusta, que hoy nos llena de orgullo y regocijo, que con la ayuda de Dios continuará avanzando siendo un faro de luz y fuente de bendición a muchos, enfrentando los retos y desafíos del presente y del futuro.

Managua, 30 de marzo del 2017. 